\title{
LVPEI Glaucoma Epidemiology and Molecular Genetic Study: teleophthalmology screening for angle-closure disease in an underserved region
}

\author{
Nikhil S. Choudhari ${ }^{1}$ Premanand Chandran ${ }^{1} \cdot$ Harsha L. Rao ${ }^{1}$ Ganesh B. Jonnadula ${ }^{1,2}$ - Uday K. Addepalli, ${ }^{1,2}$ \\ Sirisha Senthil ${ }^{1}$. Chandra Sekhar Garudadri ${ }^{1}$
}

Received: 14 March 2019 / Revised: 11 August 2019 / Accepted: 25 September 2019 / Published online: 6 November 2019

(c) The Author(s), under exclusive licence to The Royal College of Ophthalmologists 2019

\begin{abstract}
Objectives To assess the ability of teleophthalmoscopic grading of peripheral anterior chamber depth (PACD) using the van Herick $(\mathrm{vH})$ technique in detecting gonioscopically occludable angle; and to determine whether combining results from $\mathrm{vH}$ grading and ocular biometry can improve the accuracy to diagnose gonioscopically occludable angle

Methods This cross-sectional study was an offshoot of a rural population-based study, Glaucoma Epidemiology and Molecular Genetic Study (GLEAMS). A masked urban ophthalmologist graded digital slit lamp photographs of PACD by $\mathrm{vH}$ technique. Sussman four-mirror lens was used to perform dark room indentation gonioscopy. Cutoff values of the tests were, $v H$ technique: grade $\leq 2$, central anterior chamber depth (ACD), as well as axial length: $\leq 25$ th percentile and lens thickness $\geq 75$ th percentile value of the study population.

Results We studied 1965 eyes of 1029 adult participants. The vH grade was $\leq 2$ in 188 (9.5\%) eyes. The angle was occludable by gonioscopy in $101(5.1 \%)$ eyes. The performance of the $\mathrm{vH}$ test to rule out gonioscopically occludable angle was good [negative predictive value (NPV): $97.3 \%$ ], despite low sensitivity $(52.5 \%)$, while its efficacy to rule in the condition was low [positive predictive value (PPV): 28.2\%] despite high specificity (92.8\%). However, test combination strategy increased the PPV nearly twofold (53.8\%). The calculated PPV at 10\% prevalence of gonioscopically occludable angle was even higher $(70.5 \%)$.

Conclusions Van Herick technique can be incorporated into a teleophthalmology program by means of slit lamp photographs of PACD. Combined $\mathrm{vH}$ grading and ocular biometry improved the predictability of a gonioscopically occludable angle.
\end{abstract}

\section{Introduction}

Primary angle-closure glaucoma (PACG) is chronic, mostly asymptomatic and often goes undiagnosed. The prevalence of PACG is reported to be highest among people of Asian descent [1]. Besides, the prevalence of blindness due to PACG is higher compared to its counterpart primary open angle glaucoma [2-4]. The prevalence of gonioscopically

Nikhil S. Choudhari

nk1164@gmail.com

nikhil@lvpei.org

1 VST Glaucoma Center, L V Prasad Eye Institute, Dr. Kallam Anji Reddy campus, Banjara Hills, Hyderabad, India

2 Brien Holden Vision Institute, Sydney, NSW, Australia occludable angle includes the prevalence of pre-disease states. While the prevalence of PACG varied between $0.6 \%$ to $1.1 \%$ [5], the regional prevalence of gonioscopically occludable angle varied between $1.5 \%$ [6] and 14.5\% [7] in Asia. The differences in methodology between the studies may have a significant contribution to the large variation in the prevalence of gonioscopically occludable angle. Nevertheless, the disparity in utilization of health-care between urban and rural areas, and related factors, such as geographic distribution of services, accessibility, and costs involved, compound the problem [2-4].

Several models of teleophthalmology for screening, triage and remote supervision are being applied in resourceconstrained or underserved locations. Teleophthalmology can effectively transmit information on intra-ocular pressure (IOP), central corneal thickness (CCT), optic nerve head imaging and visual field examination $[8,9]$. However, the 
technology is significantly limited due to lack of angle assessment.

Gonioscopy is the reference standard for the diagnosis of eyes at risk of angle closure. However, the technique requires training, high skill and experience. Several newer tests are being considered to overcome the limitations in diagnosing angle-closure disease. These include biomicroscope-based techniques, such as the van Herick $(\mathrm{vH})$ technique [10], or newer advanced optical-based systems, like Anterior segment optical coherence tomography (ASOCT) [11-13], Scheimpflug photography [14, 15], and Scanning peripheral anterior chamber depth analyzer (SPAC) $[16,17]$.

In $\mathrm{vH}$ technique, assessment is based on a comparison of the depth of the peripheral anterior chamber to the thickness of the cornea. Compared to the optical-based systems, the $\mathrm{vH}$ technique is quick, simple, and does not require expensive and large equipment. Hence, this technique is better suited for resource-constrained or underserved locations [10]. The studies that followed the traditional $\mathrm{vH}$ technique, dark room four-mirror indentation gonioscopy and International Society Geographical and Epidemiological Ophthalmology (ISGEO) classification of primary angle-closure disease [18], achieved sensitivity between 79 and $84 \%$ and specificity between 89 and $92 \%[16,19]$. However, as the prevalence of primary angle-closure disease is low, no single diagnostic test is effective for screening.

We incorporated the $\mathrm{vH}$ technique into a novel teleophthalmology program. The program linked remote community-based eye care providers to a glaucoma specialist. The primary objective of this study was to evaluate the diagnostic performance of teleophthalmoscopic $\mathrm{vH}$ grading for the detection of gonioscopically occludable angle in a southern Indian population. The secondary objective was to combine diagnostic tests to enhance the predictability of a gonioscopically occludable angle.

\section{Materials and methods}

This observational, cross-sectional study was part of the $\mathrm{L}$ V Prasad Eye Institute's-Glaucoma Epidemiology and Molecular Genetic Study (LVPEI-GLEAMS). The LVPEIGLEAMS project aims to design models for effective screening of glaucoma at community level. Institutional Review Board approval was obtained. The detailed methodology has been described elsewhere [20]. The study center was a primary eye care center located in a remote village in the Guntur district of the state of Andhra Pradesh, Southern India. The geographic area for the study sample included 16 villages (sub-district Pedanandipadu mandal).

The LVPEI-GLEAMS data was captured between September 2010 and September 2013. Data from subjects who underwent the study procedures during the first 15 months, i.e., until November 2011, was included in this pilot study. The exclusion criteria comprises of age $<40$ years; inability to obtain good quality photographs of the peripheral anterior chamber depth (PACD), viz. corneal pathology sufficient to impede visualization of PACD and angle structures; disorganized anterior chamber; manifest strabismus; aphakic or pseudophakic status; and incomplete data collection.

A trained vision technician captured slit lamp photographs of PACD in a dark room setting with a Topcon DC3 slit lamp camera (Topcon, Bauer Drive, Oakland, NJ). A thin-slit beam was focused just within the temporal limbus, offset by 60 degrees. While taking the photograph, the participants were asked to fixate at a distant target and keep their gaze straight. The digital photographs were saved on a compact disc and sent to a masked ophthalmologist (PC) at a tertiary care center for $\mathrm{vH}$ grading. A subset of 100 photographs were randomly chosen and re-graded by the same observer on another day to determine intra-observer agreement. The same subset of photographs was also graded by a masked study optometrist (GBJ) at the study center to determine inter-observer agreement in photographic grading of the eyes by the $\mathrm{vH}$ technique. The study optometrists (GBJ, UKA) worked in a glaucoma clinic at a tertiary care center for $>7$ years and have been involved in performing all diagnostic procedures in glaucoma. The eyes were graded into $\mathrm{vH}$ grades as follows: Grade 1 was PACD $<1 / 4$ corneal thickness (CT); Grade 2 was PACD equal to $1 / 4$ CT; Grade 3 was PACD 1/4-1/2 CT; and Grade 4 was PACD equal to CT [10]. Eyes having vH grades 2 or less were considered to have narrow angles typical of angleclosure disease and eyes having $\mathrm{vH}$ grade 3 and 4 were considered to have open angles.

Gonioscopy was performed on all eyes by one of the two study optometrists using Sussman four-mirror lens under dim illumination, and making sure that the beam does not fall on the pupil. The posterior most structure visible in four quadrants was documented. Indentation was performed in participants with narrow angles, and the presence of blotchy pigments and synechiae were documented. An occludable angle was defined as non-visualization of the posterior trabecular meshwork $\geq 180$ degrees under dark room gonioscopy. We followed International Society for Geographical \& Epidemiological Ophthalmology (ISGEO) classification to label participants as primary angle-closure suspect (PACS), primary angle closure (PAC), and primary angle-closure glaucoma (PACG) [18].

A trained vision technician measured central anterior chamber depth (ACD), lens thickness (LT), and axial length (AL), using ultrasonic pachymeter AL-1000 (Tomey, Japan). The definition of abnormality for ocular biometry parameters was $\leq 25$ th percentile value for the central ACD, as well as AL and $\geq 75$ th percentile value for the LT. The 
sequence of tests is described in the methodology of the LVPEI-GLEAMS [20].

\section{Statistical analysis}

Weighted Kappa was used for intra- and inter-observer variability. Sensitivity, specificity, and positive (PPV) and negative predictive values (NPV) were used to assess the ability of $\mathrm{vH}$ technique to detect occludable angles. A multivariate model was used to evaluate the predictive ability of $\mathrm{vH}$ technique and ocular biometry. As measurements from both eyes of the same participant are likely to be correlated, bias corrected standard error was calculated during all estimations [21]. The predictive values were also calculated at $10 \%$ prevalence of occludable angle. Statistical analyses were performed using statistical software Stata 12.1 (StataCorp, College Station, TX).

\section{Results}

A total of 1217 participants underwent examination from September 2010 to November 2011. Incomplete data from 188 participants was excluded. Thus, 1029 participants, 621 $(60.3 \%)$ female and 408 (39.6\%) male, were included in the current study. The median (1st and 3rd quartiles) age of the participants was $50(45,60)$ years. We included both eyes of 936 participants and one eye of 93 participants (total number of eyes: 1965). The reasons for exclusion of fellow eyes of 93 participants were pseudophakia/ aphakia in 59 eyes, pterygium in three eyes, corneal scar in two eyes, phthisis bulbi in one eye, strabismus in one eye, and inadequate quality of slit lamp photograph in 27 eyes.

The kappa value [95\% confidence interval (CI)] of the intra- and inter-observer agreement in grading the slit lamp photographs by $\mathrm{vH}$ technique was $0.76(0.65$ to 0.87$)$ and 0.56 (0.45 to 0.67 ), respectively.

The number of eyes categorized into $\mathrm{vH}$ grade 1 to 4 were 49 (2.4\%), 139 (7\%), $908(46.2 \%)$ and 869 (44.2\%), respectively. Thus, by $\mathrm{vH}$ grading, $188(9.5 \%)$ eyes had occludable angle and the remaining 1777 (90.4\%) eyes had open angle. The angle was occludable by gonioscopy in 101 (5.1\%) eyes and was open in 1864 (94.9\%) eyes. Among 101 eyes with gonioscopically occludable angle, 56 eyes were PACS, 39 eyes were PAC, and 6 eyes were PACG. The proportion of the study eyes having PACS, PAC and PACG was $2.84,1.98$, and 0.3 , respectively.

The median (1st and 3rd quartiles) central ACD was 3.13 $(2.89,3.38) \mathrm{mm}$; the lens thickness was $4.43(4.18,4.67)$ $\mathrm{mm}$; and axial length was $22.6(22.08,23.07) \mathrm{mm}$.

The sensitivity $(95 \% \mathrm{CI})$ of $\mathrm{vH}$ classification to detect occludable angle was $52.5 \%$ (42.3 to 62.5 ) and specificity was $92.8 \%$ (91.5 to 93.9 ). The positive and negative predictive values $(95 \% \mathrm{CI})$ at $5.1 \%$ prevalence of gonioscopically occludable angle were $28.2 \%$ (21.9 to 35.2$)$ and $97.3 \%$ (96.4 to 98 ), respectively.

The correlations were poor between central ACD and LT $\left(r^{2}<0.01\right)$, central ACD and AL $\left(r^{2}=0.04\right)$, and LT and $\operatorname{AL}\left(r^{2}=0.01\right)$.

A univariate logistic regression model identified gender, central ACD, LT, AL, and vH grade (excluding age), to be significantly associated with the classification of the angle by gonioscope. This robust model took into account the correlation between two eyes of a participant. In a multivariate regression analysis, which included the significant predictors in univariate analysis, central ACD and LT, along with $\mathrm{vH}$ classification were significantly associated with the classification of the angle by gonioscopy (Table 1).

The diagnostic accuracy of the test combination strategy to rule in or rule out gonioscopically occludable angle is shown in Tables 2 and 3, respectively. The diagnostic accuracy measures of $\mathrm{vH}$ technique to detect gonioscopically occludable angle were improved by the test combination strategy.

\section{Discussion}

The aim of screening for angle-closure disease is to prevent irreversible blindness due to angle-closure glaucoma. This
Table 1 Logistic regression report for diagnosis of gonioscopically occludable angle

\begin{tabular}{|c|c|c|c|c|}
\hline \multirow[t]{2}{*}{ Variable } & \multicolumn{2}{|l|}{ Univariate regression } & \multicolumn{2}{|l|}{ Multivariate regression } \\
\hline & Odds ratio $(95 \% \mathrm{CI})$ & $P$-value & Odds ratio $(95 \% \mathrm{CI})$ & $P$-value \\
\hline Age & $1.01(0.98,1.04)$ & 0.35 & - & - \\
\hline Gender & $0.32(0.15,0.65)$ & $<0.01$ & $0.64(0.30,1.37)$ & 0.25 \\
\hline $\mathrm{vH}$ grading & $14.14(8.26,24.19)$ & $<0.01$ & $6.94(3.89,12.37)$ & $<0.01$ \\
\hline Central ACD & $0.03(0.00,0.13)$ & $<0.01$ & $0.10(0.02,0.57)$ & $<0.01$ \\
\hline Lens thickness & $2.21(1.53,3.20)$ & $<0.01$ & $1.93(1.06,3.50)$ & $\mathbf{0 . 0 3}$ \\
\hline Axial length & $0.37(0.25,0.53)$ & $<0.01$ & $0.70(0.43,1.12)$ & 0.14 \\
\hline
\end{tabular}

$C I$ confidence interval, $v H$ van Herick, $A C D$ anterior chamber depth

Statistically significant $p<0.01$ values are in bold 
Table 2 Diagnostic accuracy of the test combination strategy to rule in gonioscopically occludable angle

\begin{tabular}{lllll}
\hline Parameter & Specificity & PPV at 5.1\% prevalence & PPV at $10 \%$ prevalence & LR positive \\
\hline vH classification & $92.8(91.5,93.9)$ & $28.2(21.9,35.2)$ & $44.6(38.6,50.7)$ & $7.25(5.66,9.27)$ \\
ACD $\leq 1$ st quartile $(2.89 \mathrm{~mm})$ & $77.9(75.9,79.8)$ & $15.2(12.1,18.7)$ & $26.9(24.2,29.9)$ & $3.31(2.87,3.83)$ \\
Lens thickness $\geq$ 3rd quartile $(4.67 \mathrm{~mm})$ & $75.1(73.1,77.1)$ & $10.6(8.0,13.6)$ & $19.6(16.7,22.8)$ & $2.19(1.80,2.66)$ \\
Combined vH classification OR ACD & $97.1(96.2,97.8)$ & $44.9(34.8,55.3)$ & $62.6(54.2,70.2)$ & $15(10.7,21.2)$ \\
$\begin{array}{l}\text { Combined vH classification OR ACD OR lens } \\
\text { thickness }\end{array}$ & $98.7(98.1,99.2)$ & $53.8(39.5,67.8)$ & $70.5(59,79.9)$ & $21.5(13,35.8)$ \\
\hline
\end{tabular}

Data is presented as percentage (95\% confidence interval)

$P P V$ positive predictive value, $L R$ likelihood ratio, $v H$ van Herick, $A C D$ anterior chamber depth

Table 3 Diagnostic accuracy of the test combination strategy to rule out gonioscopically occludable angle

\begin{tabular}{lllll}
\hline Parameter & Sensitivity & NPV at $5.1 \%$ prevalence & NPV at $10 \%$ prevalence & LR negative \\
\hline vH Classification & $52.5(42.3,62.5)$ & $97.3(96.4,98)$ & $94.6(93.5,95.6)$ & $0.51(0.41,0.62)$ \\
ACD $\leq 1$ st quartile $(2.89 \mathrm{~mm})$ & $73.3(63.5,81.6)$ & $98.2(97.4,98.8)$ & $96.3(95,97.3)$ & $0.34(0.24,0.47)$ \\
Lens thickness $\geq$ 3rd quartile $(4.67 \mathrm{~mm})$ & $54.5(44.2,64.4)$ & $96.8(95.8,97.7)$ & $93.7(92.3,94.8)$ & $0.60(0.48,0.75)$ \\
Combined vH classification AND ACD & $82.2(73.3,89.1)$ & $98.7(98,99.2)$ & $97.4(96.1,98.3)$ & $0.24(0.15,0.36)$ \\
Combined vH classification AND ACD & $88.1(80.2,93.7)$ & $98.9(98.1,99.4)$ & $97.8(96.3,98.7)$ & $0.20(0.11,0.34)$ \\
AND lens thickness & & & & \\
\hline
\end{tabular}

Data is presented as percentage (95\% confidence interval)

$N P V$ negative predictive value, $L R$ likelihood ratio, $v H$ van Herick, $A C D$ anterior chamber depth

requires detecting a gonioscopically occludable angle and offering prophylaxis or treatment, as appropriate. However, a false-positive screening test may lead to inappropriate referral, anxiety to the individual, and unnecessary burden on the health-care system.

Teleophthalmology models typically involve local health-care personnel capturing images and transmitting these to ophthalmologist for assessment. It allows nonophthalmologists to screen for the disease in a short time, and makes specialist care more accessible at low cost $[8,9]$. Glaucoma screening using optic nerve head examination yielded a summary estimate $(95 \% \mathrm{CI})$ of sensitivity and specificity to be $83 \%(77,88)$ and $79 \%(66,87)$, respectively from a meta-analysis [22]. Intra-ocular pressure measurement, central corneal thickness, ACD, and visual field examination were also incorporated into teleophthalmology [8, 9, 23, 24]. However, teleophthalmic screening for angle-closure glaucoma is mostly limited by angle assessment as gonioscopy cannot be incorporated into the system. On this background, our study reports on the feasibility of incorporating $\mathrm{vH}$ grading as an angle assessment tool in teleophthalmology.

Predictive values are useful in determining the value of a test to a clinician but are influenced by the disease prevalence [25]. At the known prevalence of primary angleclosure disease in the general population, no single diagnostic test is effective for screening. At $5.1 \%$ prevalence of occludable angle in this study, only about one in three subjects with positive $\mathrm{vH}$ test result are likely to be identified with occludable angle (PPV 28.2\%, Table 2). Nevertheless, the test combination strategy shows promise and could increase the PPV nearly twofold. The prediction could be even better at $10 \%$ prevalence of gonioscopically occludable angle (PPV 70.5\%, Table 2). The test combination approach is better suited and practical for resourcepoor or underserved regions than the use of newer anterior segment imaging modalities in glaucoma. However, this approach was used sparingly in the past [26].

Van Herick test has a moderate sensitivity level. One reason could be that $\mathrm{vH}$ grades 2 and 3 are not sharply differentiated. A set of standard photographs, as done in modified $\mathrm{vH}$ grading might be a solution to this problem [27]. Another reason for moderate sensitivity of the $\mathrm{vH}$ test is the clinical pattern of the disease itself. Chronic PACG with asymptomatic nature and relatively deep peripheral anterior chamber is more common than acute or sub-acute symptomatic PACG in our population [2, 3]. Nevertheless, a relatively higher NPV of the vH classification at $97 \%$ is attributable to low prevalence of the disease. Thus, $\mathrm{vH}$ classification alone might suffice to rule out primary angle-closure disease. The NPV can be improved further by ensuring negative result on central ACD ( $>1$ st quartile of the population) and LT $(<3 \mathrm{rd}$ quartile of the population).

The Chennai Glaucoma Study and the Andhra Pradesh Eye Disease Study reported on rural urban differences in the prevalence of angle closure. Both these studies were conducted in urban, as well as rural locations in India $[2,3]$. 
Fig. 1 Flow chart showing our test combination strategy to diagnose gonioscopically occludable angle (vH: van Herick, ACD: anterior chamber depth, LT: lens thickness)

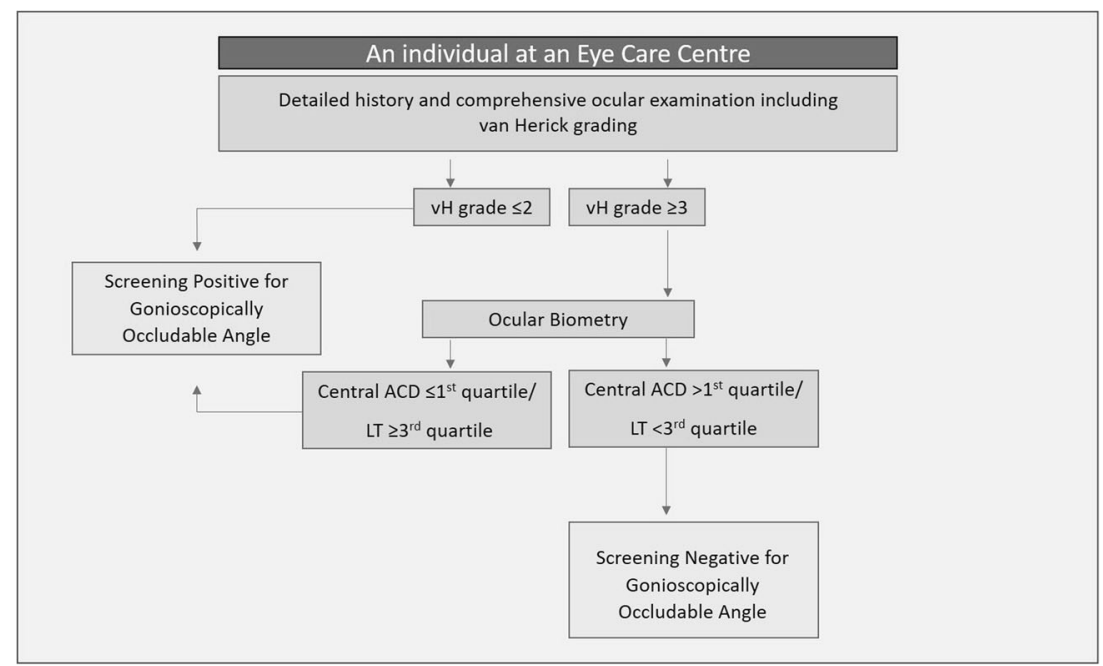

Urban and rural populations might potentially differ in many factors such as the demographic profile, disease pattern, systemic diseases, and access to ophthalmic care. Forty-five percent of the world population and nearly $60 \%$ of the African population still reside in rural areas [28]. Our study is the first to demonstrate the utility of teleophthalmology incorporated $\mathrm{vH}$ technique in a community setting. Our results show that a gonioscopically occludable angle can be identified most of the time by a combined application of $\mathrm{vH}$ technique and ocular biometry. General population-based screening programs have not been cost effective due to low prevalence of glaucoma [29]. Nevertheless, our findings have the potential to develop an opportunistic screening strategy or screening of high-risk groups for angle-closure disease at a community level.

Our study has a few limitations. The intra-observer agreement in grading the slit lamp photographs by $\mathrm{vH}$ technique was substantial and was comparable to the reported intra-observer agreement in performing $\mathrm{vH}$ technique on patients [30]. However, the inter-observer agreement in grading the slit lamp photographs by $\mathrm{vH}$ technique was only moderate. A comparison to the standard photographs while performing the $\mathrm{vH}$ technique [27] or automation of photograph-based $\mathrm{vH}$ technique are possible solutions. The latter has relevance for the more complex modification of the vH system [27]. Secondly, this study was an offshoot of a major epidemiologic study, and we could not have a single observer to perform gonioscopy of all the study participants. The two study optometrists who performed gonioscopy have over 7-year experience in the glaucoma department of a tertiary eye care center. The agreement between a reference glaucoma specialist and each of the study optometrists for gonioscopy was good on formal testing (weighted kappa 0.92 and 0.84). This was true, even when the optometrists had to categorize the gonioscopic information into open angle and various subtypes of primary angle-closure disease [31]. We believe that the inter-observer variability is likely to be reduced further with binary gonioscopic classification into "open" versus "occludable" angle as required in the current study.

In conclusion, the $\mathrm{vH}$ technique in combination with ocular biometry shows considerable potential for use in population-based or targeted or opportunistic screening for the angle-closure disease. The $\mathrm{vH}$ test can be made teleophthalmoscope-friendly by applying it to slit lamp photographs of PACD. A participant should be considered screening positive when $\mathrm{vH}$ grading is 2 or less. The remaining participants should undergo ocular biometry and those abnormal on central ACD or LT should also be considered screening positive. The remaining participants are screening negative (Fig. 1). There is $1-2 \%$ chance of missing angle-closure disease in those screened negative. Our approach showed analogous results in another study population [32]. This approach offers the advantage of using standard equipment in a primary eye care setting and shows promise for detection of primary angle-closure disease in remote regions.

\section{Summary}

\section{What was known before}

- Gonioscopy is the reference standard test to assess the angle of the anterior chamber. However, it requires high skill and is subjective.

- Van Herick Technique is a triage test in the detection of primary angle-closure disease.

- Combination of tests could improve the diagnostic accuracy of screening techniques for angle-closure 
glaucoma. However, this approach was used sparingly in the past.

\section{What this study adds}

- The van Herick (vH) technique can be made teleophthalmoscope-friendly by applying it to slit lamp photographs of peripheral anterior chamber depth.

- The vH technique in combination with ocular biometry showed considerable diagnostic accuracy in the detection of gonioscopically occludable angle.

- The test combination approach is better suited and practical for resource-poor or underserved regions than the use of newer anterior segment imaging modalities and has potential for use in population-based or targeted or opportunistic screening for the angle-closure disease.

\section{Compliance with ethical standards}

Conflict of interest HLR: Consultant to Cipla $\odot$ (Past). CSG: Consultant to Alcon $\odot$ Allergan $\odot$ (Past). The other authors declare that they have no conflict of interest.

Publisher's note Springer Nature remains neutral with regard to jurisdictional claims in published maps and institutional affiliations.

\section{References}

1. Tham YC, Li X, Wong TY, Quigley HA, Aung T, Cheng CY. Global prevalence of glaucoma and projections of glaucoma burden through 2040: a systematic review and meta-analysis. Ophthalmology. 2014;121:2081-90.

2. Vijaya L, George R, Arvind H, Baskaran M, Ve Ramesh S, Raju $\mathrm{P}$, et al. Prevalence of primary angle-closure disease in an urban south Indian population and comparison with a rural population. The Chennai Glaucoma Study. Ophthalmology. 2008;115:655-60.

3. Garudadri C, Senthil S, Khanna RC, Sannapaneni K, Rao HB. Prevalence and risk factors for primary glaucomas in adult urban and rural populations in the Andhra Pradesh Eye Disease Study. Ophthalmology. 2010;117:1352-9.

4. George R, Ve RS, Vijaya L. Glaucoma in India: estimated burden of disease. J Glaucoma. 2010;19:391-7.

5. Cho HK, Kee C. Population-based glaucoma prevalence studies in Asians. Surv Ophthalmol. 2014;59:434-47.

6. Paul C, Sengupta S, Banerjee S, Choudhury S. Angle closure glaucoma in rural and urban populations in eastern India-The Hooghly River Glaucoma Study. Indian J Ophthalmol. 2018;66:1285-90.

7. Sawaguchi S, Sakai H, Iwase A, Yamamoto T, Abe H, Tomita G, et al. Prevalence of primary angle closure and primary angleclosure glaucoma in a southwestern rural population of Japan: the Kumejima Study. Ophthalmology. 2012;119:1134-42.

8. Sreelatha OK, Ramesh SV. Teleophthalmology: improving patient outcomes? Clin Ophthalmol. 2016;10:285-95.

9. Caffery LJ, Taylor M, Gole G, Smith AC. Models of care in teleophthalmology: a scoping review. J Telemed Telecare. 2019;25:106-122
10. Van Herick W, Shaffer RN, Schwartz A. Estimation of width of angle of anterior chamber. Incidence and significance of the narrow angle. Am J Ophthalmol. 1969;68:626-9.

11. Nolan WP, See JL, Chew PT, Friedman DS, Smith SD, Radhakrishnan $\mathrm{S}$, et al. Detection of primary angle closure using anterior segment optical coherence tomography in Asian eyes. Ophthalmology. 2007;114:33-9.

12. Pekmezci M, Porco TC, Lin SC. Anterior segment optical coherence tomography as a screening tool for the assessment of the anterior segment angle. Ophthalmic Surg Lasers Imaging. 2009;40:389-98.

13. Narayanaswamy A, Sakata LM, He MG, Friedman DS, Chan YH, Lavanya $\mathrm{R}$, et al. Diagnostic performance of anterior chamber angle measurements for detecting eyes with narrow angles: an anterior segment OCT study. Arch Ophthalmol. 2010;128:1321-7.

14. Kurita N, Mayama C, Tomidokoro A, Aihara M, Araie M. Potential of the pentacam in screening for primary angle closure and primary angle closure suspect. J Glaucoma. 2009;18:506-12.

15. Hong S, Yi JH, Kang SY, Seong GJ, Kim CY. Detection of occludable angles with the Pentacam and the anterior segment optical coherence tomography. Yonsei Med J. 2009;50:525-8.

16. Baskaran M, Oen FT, Chan YH, Hoh ST, Ho CL, Kashiwagi K, et al. Comparison of the scanning peripheral anterior chamber depth analyzer and the modified van Herick grading system in the assessment of angle closure. Ophthalmology. 2007;114:501-6.

17. Zhang Y, Li SZ, Li L, Thomas R, Wang NL. The Handan Eye Study: comparison of screening methods for primary angle closure suspects in a rural Chinese population. Ophthalmic Epidemiol. 2014;21:268-75.

18. Foster PJ, Buhrmann R, Quigley HA, Johnson GJ. The definition and classification of glaucoma in prevalence surveys. Br J Ophthalmol. 2002;86:238-42.

19. Dabasia PL, Edgar DF, Murdoch IE, Lawrenson JG. Noncontact screening methods for the detection of narrow anterior chamber angles. Invest Ophthalmol Vis Sci. 2015;56:3929-35.

20. Addepalli UK, Jonnadula GB, Garudadri CS, Rao HL, Rani PK, Chakrabarti S, et al. LV Prasad Eye Institute Glaucoma Epidemiology and Molecular Genetic Study (LVPEI- GLEAMS). Report 1: study design and research methodology. Ophthalmic Epidemiol. 2013;20:188-95.

21. Glynn RJ, Rosner B. Accounting for the correlation between fellow eyes in regression analysis. Arch Ophthalmol. 1992;110:381-7.

22. Thomas SM, Jeyaraman MM, Hodge WG, Hutnik C, Costella J, Malvankar-Mehta MS. The effectiveness of teleglaucoma versus in-patient examination for glaucoma screening: a systematic review and meta-analysis. PLoS ONE. 2014;9:e113779.

23. Kumar S, Middlemiss C, Bulsara M, Guibilato A, Morgan W, Tay-Kearney ML, et al. Telemedicine-friendly, portable tonometers: an evaluation for intraocular pressure screening. J Clin Exp Ophthalmol. 2006;34:666-70.

24. Ianchulev T, Pham P, Makarov V, Francis B, Minckler D. Peristat: a computer-based perimetry self-test for cost-effective population screening of glaucoma. Curr Eye Res. 2005;30:1-6.

25. Parikh R, Mathai A, Parikh S, Chandra Sekhar G, Thomas R. Understanding and using sensitivity, specificity and predictive values. Indian J Ophthalmol. 2008;56:45-50.

26. Congdon NG, Quigley HA, Hung PT, Wang TH, Ho TC. Screening techniques for angle-closure glaucoma in rural Taiwan. Acta Ophthalmol Scand. 1996;74:113-9.

27. Foster PJ, Devereux JG, Alsbirk PH, Lee PS, Uranchimeg D, Machin D, et al. Detection of gonioscopically occludable angles and primary angle closure glaucoma by estimation of limbal chamber depth in Asians: modified grading scheme. Br J Ophthalmol. 2000;84:186-92. 
28. Data on Annual Percentage of Population at Mid-Year Residing in Urban Areas. [UNITED NATIONS web site] Available at: http:// esa.un.org/unpd/wup/dataquery/. Accessed February 2, 2019.

29. McManus JR, Netland PA. Screening for glaucoma: rationale and strategies. Curr Opin Ophthalmol. 2013;24:144-9.

30. Choudhari NS, Chanda S, Khanna R, Senthil S, Garudadri CS. Diagnostic accuracy of van Herick technique to detect pre-disease states of primary angle closure glaucoma in a resource constraint region. Ophthalmic Epidemiol. 2019;26:175-182.
31. Addepalli U, Jonnadula GB, Garudadri CS, Rao HL, Senthil S, Papas EB, et al. Agreement of glaucoma specialists and experienced optometrists in gonioscopy and optic disc evaluation. $\mathbf{J}$ Optom. 2013;6:212-8.

32. Choudhari NS, George R, Asokan R, Khanna R, Vijaya L, Garudadri CS. Combination of Simple diagnostic tests to detect primary angle closure disease in a resource-constrained region. Ophthalmic Epidemiol. 2019 Aug 7:1-9. doi: 10.1080/ 09286586.2019.1650380. [Epub ahead of print] 